\title{
Functional interactions between type IV secretion systems involved in DNA transfer and virulence
}

\section{Correspondence \\ Matxalen Llosa \\ Ilosam@unican.es}

Received 4 August 2005

Revised 12 September 2005

Accepted 15 September 2005

\author{
Héctor D. de Paz, ${ }^{1}$ Félix J. Sangari, ${ }^{1}$ Silvia Bolland, ${ }^{1} \dagger$ \\ Juan M. García-Lobo, ${ }^{1}$ Christoph Dehio, ${ }^{2}$ Fernando de la Cruz $^{1}$ \\ and Matxalen Llosa ${ }^{1}$ \\ ${ }^{1}$ Departamento de Biología Molecular (Unidad Asociada al CIB-CSIC), Universidad de \\ Cantabria, C. Herrera Oria s/n, 39011 Santander, Spain \\ ${ }^{2}$ Division of Molecular Microbiology, Biozentrum of the University of Basel, Klingelbergstrasse \\ 70, $\mathrm{CH}-4056$ Basel, Switzerland
}

\begin{abstract}
This paper reports an analysis of the functional interactions between type IV secretion systems (T4SS) that are part of the conjugative machinery for horizontal DNA transfer (cT4SS), and T4SS involved in bacterial pathogenicity (pT4SS). The authors' previous work showed that a conjugative coupling protein (T4CP) interacts with the VirB10-type component of the T4SS in order to recruit the protein-DNA complex to the transporter for conjugative DNA transfer. This study now shows by two-hybrid analysis that conjugative T4CPs also interact with the VirB10 element of the pT4SS of Agrobacterium tumefaciens ( $A t$ ), Bartonella tribocorum (Bt) and Brucella suis (Bs). Moreover, the VirB10 component of a cT4SS (protein TrwE of plasmid R388) could be partially substituted by that of a pT4SS (protein TrwE of $B t$ ) for conjugation. This result opens the way for the construction of hybrid T4SS that deliver DNA into animal cells. Interestingly, in the presence of part of the Bs T4SS the R388 T4SS protein levels were decreased and R388 conjugation was strongly inhibited. Complementation assays between the Trw systems of R388 and $B t$ showed that only individual components from the so-called 'core complex' could be exchanged, supporting the concept that this core is the common scaffold for the transport apparatus while the other 'peripheral components' are largely system-specific.
\end{abstract}

\section{INTRODUCTION}

Type IV secretion systems (T4SS) constitute a family of macromolecular transporters involved in bacterial functions as different as conjugative DNA transfer and virulence (Cascales \& Christie, 2003). Conjugative T4SS (cT4SS) are part of the bacterial machinery involved in horizontal DNA transfer among bacteria. Pathogenic T4SS (pT4SS) are required by many pathogens to infect their eukaryotic hosts; in many instances they have been shown to be the transport channels to eukaryotic cells of essential virulence factors for establishment of the donor bacterium in the eukaryotic host. In the case of Agrobacterium tumefaciens (At), its T4SS can be considered a cT4SS as well as a pT4SS, since it is required for T-DNA transfer into the plant host cell.

In spite of the differences between cT4SS and pT4SS

†Present address: Laboratory of Immunogenetics, NIAID/NIH, 12441 Parklawn Drive, Rockville, MD 20852, USA.

Abbreviations: At, Agrobacterium tumefaciens; Bs, Brucella suis; Bt, Bartonella tribocorum; Ec, Escherichia coli; T4SS, type IV secretion system(s); cT4SS, T4SS involved in bacterial conjugation; pT4SS, T4SS involved in pathogenicity; T4CP, coupling protein. in the nature of the secreted substrate (nucleoprotein vs protein complex), in their biological role (DNA transfer vs virulence), and in the target cell (prokaryotic vs eukaryotic), there is homology among them. Most T4SS are formed by 11 proteins, named VirB1 to VirB11 for the components of the prototypical At T4SS. The overall architecture of the transporter is conserved in the family, as shown by a common gene organization (see Fig. 1), the same membrane topology of each component, and conservation of protein-protein interactions between the T4SS components. For instance the VirB7-VirB9 interaction has been described for the T4SS of At (Baron et al., 1997; Das et al., 1997; Spudich et al., 1996), Bordetella pertussis (Farizo et al., 1996), Bartonella henselae (Shamaei-Tousi et al., 2004) and Xanthomonas axonopodis (Alegria et al., 2005).

Several lines of evidence suggest that there is a 'core' complex made up of proteins VirB7, VirB8, VirB9 and VirB10 that would make up the central transmembrane channel. Topology studies indicate that these proteins are located mostly in the periplasm. VirB8, VirB9 and VirB10 were shown to interact with each other (Beaupre et al., 1997; Das \& Xie, 2000), in addition to the VirB7-VirB9 interaction already mentioned. All four proteins can be extracted 

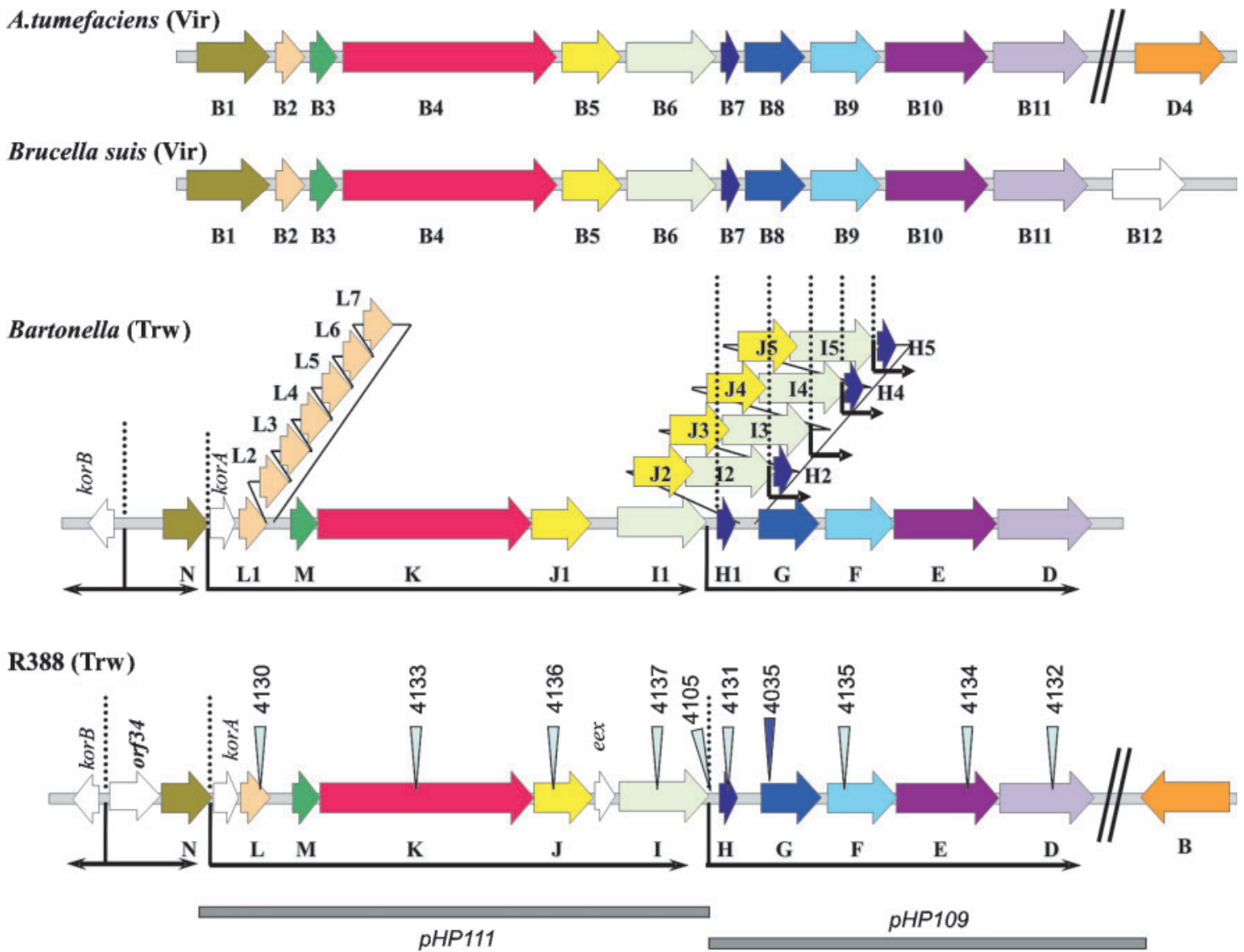

Fig. 1. Scheme of T4SS genetic determinants. Representation of the gene arrays that code for the T4SS studied in this work: At-Vir (Berger \& Christie, 1994), Bs-VirB (O'Callaghan et al., 1999), Bt-Trw (Seubert et al., 2003) and R388-Trw (this work). Homologous genes are represented in the same colour. Dashed lines represent genetic discontinuities. The prefix of the genes (vir or trw, as indicated) has been omitted for clarity. In the maps of R388 and Bt T4SS, postulated transcriptional units are indicated with black arrows that start at the proposed promoter sites and point in the direction of transcription. kor boxes are indicated by vertical dashed lines. In the Bt-Trw region, the seven trw $L$ repetitions and the five trwJ-trwl-trwH repetitions are designated as in Seubert et al. (2003). The R388 insertion mutants used in this work are indicated with triangles at the site of the transposon insertion and the name of the plasmid, without the pSU prefix. The dark triangle indicates the $\Omega$ insertion in pSU4035. Grey rectangles show the extent of the trw fragments cloned in plasmids pHP109 and pHP111.

from the membrane as a complex (Krall et al., 2002). Furthermore, there is functional dependence of one protein upon another: VirB9 and VirB10 form clusters in the bacterial envelope only if VirB8 is present (Kumar et al., 2000), VirB8 and VirB9 sequentially bind the substrate DNA during its exit pathway through the At T4SS (Cascales \& Christie, 2004a), VirB10 undergoes an energy-dependent conformational change to interact with the VirB7-VirB9 complex (Cascales \& Christie, 2004b), and VirB9-VirB10 complex formation is required for substrate passage through the secretion channel (Cascales \& Christie, 2004a, b).

The other components that make up a T4SS can be summarized as follows (for a review and update, see Cascales \& Christie, 2003; Llosa \& O'Callaghan, 2004): two highly conserved inner-membrane-bound NTPases, VirB11 and VirB4, are involved in early substrate transfer reactions
(Atmakuri et al., 2004); VirB2 and VirB5 are pilus components (Lai \& Kado, 1998; Schmidt-Eisenlohr et al., 1999); VirB6 is an integral membrane protein required for T4SS assembly and function (Hapfelmeier et al., 2000; Jakubowski et al., 2004); VirB1 is a lytic transglycosylase required for T4SS early assembly (Koraimann, 2003), although not an essential component in all systems; finally, the function of the outer-membrane associated protein VirB3 is unknown.

Many T4SS, including all cT4SS, have an associated coupling protein (T4CP). T4CPs are proteins anchored to the inner membrane through their $\mathrm{N}$-terminus, so named because they interact both with the secretion substrate and with the secretion machinery (Cabezón et al., 1997; Llosa et al., 2003). Besides their coupling role, they may act as DNA pumps during conjugation (Gomis-Rüth et al., 2001; 
Tato et al., 2005). Specific protein-protein interactions between the T4CP and cT4SS components have now been described. TrwB, the T4CP of conjugative plasmid R388, interacts with proteins $\mathrm{TrwC}$ and TrwA, which bind to the substrate DNA, and with protein TrwE, a VirB10 homologue (Llosa et al., 2003). Moreover, T4CPs interact with VirB10 homologues from heterologous cT4SS so that they can deliver their substrate DNA through the heterologous transporter, with efficiencies that correlate with the strength of the corresponding T4CP-VirB10 interaction (Llosa et al., 2003). Interactions of the At T4CP have been described with a protein substrate, protein VirE2, and with the T4SS NTPases VirB4 and VirB11 (Atmakuri et al., 2003, 2004).

Functional complementation between homologues from different T4SS has been reported in a few instances: the VirB5 homologues of two cT4SS, TraC protein of conjugative plasmid pKM101 and VirB5 of At T4SS (Schmidt-Eisenlohr et al., 1999); and some VirB1 homologues, but not others, could be exchanged (Hoppner et al., 2004). The most related T4SS systems described to date are the Trw systems found in the conjugative plasmid R388 and in Bartonella spp. [B. henselae and B. tribocorum (Bt)], which are a cT4SS and a pT4SS respectively. Identities among the Trw components of each system range from 25 to $80 \%$; the 'core' components share more than $50 \%$ identity. Functional complementation was observed between the TrwD and TrwH components of both systems (Seubert et al., 2003), underscoring the close relationships between T4SS even when their biological role involves very different processes. Thus, the Trw T4SS of R388 and Bartonella spp. are probably the best candidates to obtain a hybrid c/pT4SS which ideally could be used to deliver DNA into the eukaryotic host cells (Llosa \& de la Cruz, 2005).

In this work we undertook an extended analysis of T4CPT4SS interactions and showed that T4CPs also interact with the VirB10-like component of several pT4SS. This interaction reflects a functional interaction in the case of the Trw T4SS, as shown by functional complementation between the respective T4SS components. We performed a complementation analysis between these two systems in order to obtain information about the building blocks of a T4SS that can be exchanged. Our results support the concept of a 'core complex' of highly conserved components that can be substituted, while the 'peripheral' components are more specific for their host/function.

\section{METHODS}

Bacterial strains. These are listed in Table 1. The Escherichia coli (Ec) lac $^{\mathrm{q}}$ strain D1210 was used for Plac-driven expression. For conjugation experiments, strains D1210, HB101 or DH5 $\alpha$ were used as donor and recipient strains, as indicated. Strain DHM1 was used as a host in two-hybrid assays. Strain JC7623 was used to transfer mutations to R388 by homologous recombination. At genomic DNA was obtained from wild-type nopaline At strain C58. Brucella suis (Bs) 1330 is a virulent wild-type strain. Bs 1330 virB5::Kan is a mini-Tn5-km2 virB5 mutant.

Table 1. Bacterial strains

\begin{tabular}{|c|c|c|}
\hline Strain & Genotype $^{*}$ & Source or reference \\
\hline \multicolumn{3}{|l|}{$\begin{array}{l}\text { Agrobacterium } \\
\text { tumefaciens }(A t)\end{array}$} \\
\hline C58 & Wild-type nopaline & $\begin{array}{l}\text { Patricia Zambryski, University } \\
\text { of California at Berkeley }\end{array}$ \\
\hline \multicolumn{3}{|l|}{ Brucella suis (Bs) } \\
\hline 1330 & Wild-type & David O'Callaghan, INSERM, France \\
\hline $\begin{array}{l}1330 \text { virB5: : Kan } \\
\text { Escherichia coli }(E c)\end{array}$ & $\mathrm{Km}^{\mathrm{R}}$ virB5 polar mutant & O'Callaghan et al. (1999) \\
\hline D1210 & $\begin{array}{l}\mathrm{Sm}^{\mathrm{R}} \text { proA2 leuB6 thi-1 ara-14 lacY1 } \\
\text { galK2 xyl5 mtl-1 recA13 hsdR hsdM } \\
\text { rpsL lacl }{ }^{\mathrm{q}}\end{array}$ & Sadler et al. (1980) \\
\hline $\mathrm{DH} 5 \alpha$ & 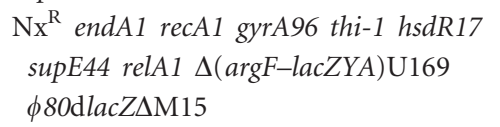 & Grant et al. (1990) \\
\hline DHM1 & $\begin{array}{l}\mathrm{Nx}^{\mathrm{R}} \text { cya-854 recA1 endA1 gyrA96 thi-1 } \\
\text { hsdR17 spoT1 rfbD1 glnV44(AS) }\end{array}$ & Karimova et al. (2005) \\
\hline HB101 & $\begin{array}{l}\mathrm{Sm}^{\mathrm{R}} \text { thi-1 hsdS20 }\left(\mathrm{r}_{\mathrm{B}}^{-} \mathrm{m}_{\mathrm{B}}^{-}\right) \text {recA13 } \\
\text { ara-14 leuB6 proA2 lacY1 galK2 } \\
\text { rpsL20 xyl-5 mtl-1 supE44 }\end{array}$ & Boyer \& Roulland-Dussoix (1969) \\
\hline JC7623 & $\begin{array}{l}\mathrm{Sm}^{\mathrm{R}} \text { recB21 recC22 sbcB15 sbcC arg } \\
\text { thi thr leu pro his strA }\end{array}$ & Kushner et al. (1971) \\
\hline
\end{tabular}

${ }^{\star} \mathrm{Km}^{\mathrm{R}}, \mathrm{Sm}^{\mathrm{R}}$ and $\mathrm{Nx}^{\mathrm{R}}$, resistant to kanamycin, streptomycin and nalidixic acid, respectively. 
Table 2. Plasmids

\begin{tabular}{|c|c|c|}
\hline Plasmid & Description & Reference \\
\hline pAB2 & pLAFR5-Km:: Bt trw region & Seubert et al. (2003) \\
\hline pFJS134 & pRL662:: MobW (oriT+trwABC) & This work \\
\hline pFJS193 & pRL662::Mob (CloDF13) & This work \\
\hline pHG327 & $\mathrm{Ap}^{\mathrm{R}}$ Cloning vector, Rep (pMB1) & Stewart et al. (1986) \\
\hline pHG329 & $\mathrm{Ap}^{\mathrm{R}}$ Cloning vector, Rep (pMB1) & Stewart et al. (1986) \\
\hline pHP100 & pSU24:: trwE $(B t)$ & This work \\
\hline pHP101 & pSU24:: virB10 (Bs) & This work \\
\hline pHP102 & pSU24:: trwE (R388) & This work \\
\hline pHP109 & pSU18:: trwH-D+korB & This work \\
\hline pHP111 & pHG329:: trwL-I & This work \\
\hline pMTX513 & pUT18C: : trwB & Llosa et al. (2003) \\
\hline pMTX514 & pT25:: $\operatorname{trw} B$ & Llosa et al. (2003) \\
\hline pMTX631 & pUT18C: : trwE & Llosa et al. (2003) \\
\hline pMTX632 & pT25:: trwE & Llosa et al. (2003) \\
\hline pMTX644 & pUT18C : : traJ & Llosa et al. (2003) \\
\hline pMTX651 & pT25:: traJ & Llosa et al. (2003) \\
\hline pMTX667 & pUT18C: : traF & Llosa et al. (2003) \\
\hline pMTX671 & pUT18C: : virB10 (At) & This work \\
\hline pMTX672 & pUT18C : : virD4 (At) & This work \\
\hline pMTX673 & pT25::virB10 $(A t)$ & This work \\
\hline pMTX674 & pT25:: traF & Llosa et al. (2003) \\
\hline pMTX677 & pUT18C: : pilX10 & Llosa et al. (2003) \\
\hline pMTX679 & pUT18C: : $\operatorname{tax} B$ & Llosa et al. (2003) \\
\hline pMTX680 & pT25:: $\operatorname{tax} B$ & Llosa et al. (2003) \\
\hline pMTX682 & pT25:: pilX10 & This work \\
\hline pMTX687 & pT25:: virD4 $(A t)$ & This work \\
\hline pMTX688 & pT25:: virB10 (Bs) & This work \\
\hline pMTX689 & pUT18C: : virB10 (Bs) & This work \\
\hline pMTX697 & pUT18C: : trwE $(B t)$ & This work \\
\hline pMTX698 & pT25:: trwE $(B t)$ & This work \\
\hline pRL662 & $\mathrm{Gm}^{\mathrm{R}}$ broad host-range cloning vector & Vergunst et al. (2000) \\
\hline pSU18 & $\mathrm{Cm}^{\mathrm{R}}$ cloning vector, Rep (p15A) & Bartolomé et al. (1991) \\
\hline pSU24 & $\mathrm{Cm}^{\mathrm{R}}$ cloning vector, Rep (p15A) & Bartolomé et al. (1991) \\
\hline pSU1423 & pSU18:: oriT + trwABC & Bolland et al. (1990) \\
\hline pSU1425 & R388 without EcoRI site & Llosa et al. (1994) \\
\hline pSU2007 & $\mathrm{R} 388 \mathrm{Km}^{\mathrm{R}}$ & Martínez \& de la Cruz (1988) \\
\hline pSU1087 & pUB2413 $\left(\mathrm{Cm}^{\mathrm{R}}, \operatorname{Rep}(\mathrm{p} 15 \mathrm{~A}):: \operatorname{trwA}-\operatorname{trwN}\right.$ & Bolland et al. (1990) \\
\hline pSU4035 & pSU1087:: $\Omega$ insertion in $\operatorname{trw} G$ & Bolland et al. (1990) \\
\hline pSU4051 & pHG327:: oriT + trwABC & Bolland et al. (1990) \\
\hline pSU4058 & pHG327:: trwL-trwD & Bolland et al. (1990) \\
\hline pSU4063 & pSU $4058:: \operatorname{Tn} 5 \operatorname{tac} 1$ in $\operatorname{trwD}$ & This work (Table 4 ) \\
\hline pSU4064 & pSU4058::Tn5tac1 in trwK & This work (Table 4) \\
\hline pSU4065 & pSU4058:: Tn5tac1 in trwL & This work (Table 4 ) \\
\hline pSU4067 & pSU4058:: Tn5tac1 in trwE & This work (Table 4 ) \\
\hline pSU4070 & pSU4058:: Tn5tacl in trwF & This work (Table 4) \\
\hline pSU4078 & pSU4058::Tn5tac1 in trwH & This work (Table 4) \\
\hline pSU4082 & pSU4058:: Tn5tac1 in trwJ & This work (Table 4) \\
\hline pSU4089 & pSU4058:: Tn5tac1 in trwI & This work (Table 4) \\
\hline pSU4105 & pSU4058::Tn5tac1 between trwH and trwI & This work \\
\hline pSU4130 & pSU1425::Tn5tac1 in trwL & This work (Table 4) \\
\hline pSU4131 & pSU1425::Tn5tac1 in trwH & Seubert et al. (2003) \\
\hline pSU4132 & pSU1425::Tn5tac1 in trwD & Rivas et al. (1997) \\
\hline pSU4133 & pSU1425:: Tn5tac1 in $\operatorname{trwK}$ & Llosa et al. (2003) \\
\hline pSU4134 & pSU1425::Tn5tac1 in trwE & Llosa et al. (2003) \\
\hline
\end{tabular}


Table 2. cont.

\begin{tabular}{|c|c|c|}
\hline Plasmid & Description & Reference \\
\hline pSU4135 & pSU1425::Tn5tac1 in trwF & This work (Table 4) \\
\hline pSU4136 & pSU1425:: Tn5tac1 in trwJ & This work (Table 4) \\
\hline pSU4137 & pSU1425:: Tn5tac1 in trwI & This work (Table 4) \\
\hline pT25 & $\mathrm{Cm}^{\mathrm{R}}$ vector for $\mathrm{T} 25$ fusions, Rep (p15A) & Karimova et al. (1998) \\
\hline pT25zip & Positive control for two-hybrid assays & Karimova et al. (1998) \\
\hline pUT18C & $A p^{R}$ vector for $T 18$ fusions, Rep (pMB1) & Karimova et al. (2001) \\
\hline pUT18Czip & Positive control for two-hybrid assays & Karimova et al. (2001) \\
\hline
\end{tabular}

Plasmid constructions. Plasmids used are shown in Table 2. Plasmids were constructed by using standard recombinant DNA technology (Sambrook et al., 1989). Inserts were obtained by restriction digestion or PCR amplification with specific oligonucleotides which created the appropriate restriction sites for cloning into the same sites of the corresponding vector. All constructions involving PCR were confirmed by DNA sequencing. Table 3 shows the oligonucleotides used, with the restriction sites introduced underlined. As template DNA for amplification, we used plasmid minipreps of R388 derivatives for R388 genes, total genomic DNA obtained as described by Bruce \& Jordens (1991) for Bs genes, and miniprep DNA from cosmid pAB2 for Bt genes.

To obtain fusion proteins with the T18 and T25 domains of adenylate cyclase for two-hybrid assays, ORFs of the test proteins were placed inframe with T18 and T25 in plasmids pUT18C and pT25 using BamHI and $K p n I$ restriction sites. The only exception is At-VirD4, since the gene includes a BamHI site, so the oligonucleotides introduced instead a XbaI site (T18 fusion) or SmaI site (T25 fusion).

To produce wild-type proteins R388-TrwE (pHP102), Bt-TrwE (pHP100) and Bs-VirB10 (pHP101), the corresponding genes were amplified with oligonucleotides that introduced SalI and EcoRI sites and the digested products were inserted into the same sites of vector pSU24. Expression of the inserted genes was dependent on the vector lac promoter.

Plasmid pFJS134 was constructed by insertion of an EcoRI-HindIII fragment from pSU4051 carrying the R388 oriT-trwABC region into the same sites of broad-host-range vector pRL662. Plasmid pFJS193 contains a $3.6 \mathrm{~kb}$ fragment from plasmid pSU4814 (Núñez \& de la Cruz, 2001) cloned into the EcoRI-HindIII sites of pRL662; this fragment contains the mobilization region of CloDF13.

To obtain separately the two putative R388 trw operons present in pSU4058, we used plasmid pSU4105 (Table 2), which carries a Tn5tac1 insertion at coordinate 4101 (GenBank sequence X81123), in the intergenic region between the two proposed transcripts. The insertion lies between trwH and trwI and does not affect any trw gene, and in fact it was the only mutant obtained that retained sensitivity to the pilusspecific phage PRD1 (data not shown). Since the transposon has several restriction sites close to both ends and pSU4058 has HindIII sites at both ends of the R388 trw region, the $5.5 \mathrm{~kb}$ EcoRI-HindIII fragment and the $4 \mathrm{~kb} B g l \mathrm{II}-H i n \mathrm{dIII}$ fragment from pSU4105 contained the korA-trwI and trwH-trwD regions of R388, respectively. Plasmid pHP111 was constructed by insertion of the EcoRI-HindIII fragment

Table 3. Oligonucleotides

\begin{tabular}{|c|c|c|}
\hline Name & Used for construct & Sequence $\left(5^{\prime}-3^{\prime}\right)^{*}$ \\
\hline CYABam-B10 & pT25/pUT18C:At-virB10 & CCAGGATCCCATGAACGACGACAATCAGCA \\
\hline B10stop-Kpn & pT25/pUT18C:At-virB10 & CCAGGTACCTTATGGACGGCGATCCC \\
\hline CYAXba-D4 & pUT18C:At-virD4 & CCATCTAGAGATGAATTCGAGCAAGACTAC \\
\hline CYA-Sma-D4 & pT25:At-virD4 & CCACCCGGGTAATGAATTCGAGCAAGACTAC \\
\hline D4stop-Kpn & pT25/pUT18C:At-virD4 & CCAGGTACCTCATTTCACCGAGTGCGA \\
\hline CYABam-BarTrwE2 & pT25/pUT18C:Bt-trwE & CCAGGATCCAATGTTTGACAATAAGGAAGGAGA \\
\hline BarTrwEstop-Kpn & pT25/pUT18C:Bt-trwE & CCAGGTACCTAACGTGTGACGAGACTG \\
\hline CYABam-BruB10 & pT25/pUT18C:Bs-virB10 & CCAGGATCCAATGACACAGGAAAACATTC \\
\hline BruB10 stop-Kpn & pT25/pUT18C:Bs-virB10 & CCAGGTACCTCACTTCGGTTTGACATCA \\
\hline SalSD-trwE & pSU24:trwE & CCAGTCGACAAGGAGGCAACTAATGTTCGG \\
\hline trwEstop-Eco & pSU24:trwE & CCAGAATTCCGGTTCCCGCTACTTAGT \\
\hline SalSD-BarTrwE & pSU24:Bt-trwE & CCAGTCGACGGAGGAAAATAATGTTTGACA \\
\hline BarTrwEstop-Eco & pSU24:Bt-trwE & CCAGAATTCTCTTTTTGTATAGGGTTAAC \\
\hline SalSD-BruB10 & pSU24:Bs-virB10 & CCAGTCGACGGGGAGAACCTGCAATGAC \\
\hline BruB10stop-Eco & pSU24:Bs-virB10 & CCAGAATTCTCACTTCGGTTGGACATCA \\
\hline Eco-KorA & pSU18:korA & CCAGAATTCAAGGGCCAACCAAAATG \\
\hline KorAstop-Bam & pSU18:korA & CCAGGATCCGTTAGCTTTCGCCCGGA \\
\hline
\end{tabular}

${ }^{\star}$ New restriction sites are underlined. 
from pSU4105 into the same sites of vector pHG329. Plasmid pHP109 carries the R388 trwH-trwD operon in vector pSU18. Since this operon includes a Kil function (Bolland et al., 1990), the plasmid was constructed in two steps: first, the korA gene from R388 was PCRamplified and cloned into the EcoRI-BamHI sites of vector pSU18, selecting for the orientation that allows KorA expression from the vector lactose promoter; second, the BglII-HindIII fragment from pSU4105 was inserted into the BamHI-HindIII sites of the previous construction. These plasmids were maintained in strain D1210, which has a chromosomal lacl ${ }^{q}$ gene, to avoid toxicity by expression of the trw genes from the vector lac promoter.

Two-hybrid assay. Strain DHM1 was co-transformed with plasmids bearing a T25 and a T18 fusion. Three independent transformants were grown together overnight in liquid medium at $30^{\circ} \mathrm{C}$, then $10 \mu \mathrm{l}$ samples of these cultures were spread on sectors of $\mathrm{X}$ Gal-containing plates to observe and compare the blue colour. $\beta$ Galactosidase levels were measured on $100 \mu \mathrm{l}$ samples as described by Miller (1992). All experiments included positive and negative controls. Plasmid pSU4111 (Moncalián et al., 1997), which carries lac $Z$ under the control of the lactose promoter, produced about 6000 Miller units in this system.

Quantitative mating assays. Samples $(100 \mu \mathrm{l}$, or $1 \mathrm{ml}$ in the case of matings involving Bs strains) of overnight cultures of donor and recipient strains were mixed; cells were collected and placed on $0.22 \mu \mathrm{m}$ filters on prewarmed agar plates for $1 \mathrm{~h}$ at $37^{\circ} \mathrm{C}$. When indicated, IPTG $(0.5 \mathrm{mM})$ was added to the agar plate. To induce expression of Bs virB genes by low $\mathrm{pH}$ (Boschiroli et al., 2002), the $B s$ pellets were resuspended in $1 \mathrm{ml} \mathrm{MM}$ broth at $\mathrm{pH} 4 \cdot 5$ (Rouot et al., 2003), and incubated for $4 \mathrm{~h}$ at $37^{\circ} \mathrm{C}$ with shaking prior to mating. Plating was done in selective medium for both donor cells and transconjugants. Transfer frequencies are expressed as the number of transconjugants per donor cell. The data reported are the mean of at least two independent assays. Mean values were calculated as simple means of the logarithm of the frequencies obtained, followed by calculation of the anti-logarithm of this mean value.

Transposon mutagenesis. In vivo insertional mutagenesis with transposon Tn5tacl was carried out as described by Chow \& Berg (1988) on plasmid pSU4058. Selected transposon insertions were then transferred to plasmid pSU1425 by homologous recombination using strain JC7623, as previously described (Llosa et al., 1994).

Bacteriophage PRD1 sensitivity assays. Sensitivity to the pilusspecific phage PRD1 was assayed as previously described (Bolland et al., 1990).

Cell extracts for protein analysis. Bacterial cultures $(50 \mathrm{ml})$ in early stationary phase were harvested at 4500 r.p.m. for 15 min. Bacterial pellets were resuspended in $500 \mu$ PBS buffer containing $1 \mathrm{mM}$ EDTA, $1 \mathrm{mM}$ PMSF and $10 \mathrm{mM}$ benzamidine. The suspensions were transferred to chilled FASTPREP tubes containing glass beads (lysing matrix B) and cells lysed in a FASTPREP FP120 instrument (Bio 101 Thermo Savant) at a speed of 6.0 for two cycles of $30 \mathrm{~s}$. Cell supernatants were harvested by centrifugation at 13000 r.p.m. for $15 \mathrm{~min}$ at $4{ }^{\circ} \mathrm{C}$. Protein concentrations were quantified by the Bradford method using decimal dilutions of BSA as a standard, and kept frozen at $-80^{\circ} \mathrm{C}$.

Immunoblot analysis. Samples $(20 \mu \mathrm{g})$ of total protein from each of the $B s$ cell lysates were run in $12 \%(w / v)$ SDS-PAGE. After the run, protein samples were transferred from the SDS-PAGE gel to a PVDF membrane (Bio-Rad) (Towbin et al., 1979), and blocked using $3 \%(\mathrm{w} / \mathrm{v})$ bovine serum albumin (Sigma) in TBST. Incubation with primary antibody was performed for $1 \mathrm{~h}$ at room temperature using rabbit polyclonal antibodies at the following dilutions: anti-TrwC (Grandoso et al., 1994), 1:10000; anti-TrwD
(Rivas et al., 1997), 1:5000; anti-TrwF (Sastre, 1996; Seubert et al., 2003), 1:20000. The secondary antibody was goat anti-rabbit IgG horseradish peroxidase conjugate $(1: 2000$ dilution) (Pierce). Blots were then developed with SuperSignal West Dura Extended Duration Substrate (Pierce), and either exposed to Hyperfilm MP (Amersham), or quantified by using the Chemidoc system and Quantity One software (Bio-Rad).

\section{RESULTS}

\section{Protein-protein interactions between T4CP and pT4SS}

Previous work showed that the strength of a T4CP-VirB10like interaction correlates with the ability of a given cT4SS to serve as a conduit for the heterologous substrates driven by the T4CP (Llosa et al., 2003). Using the bacterial two-hybrid assay (Karimova et al., 1998), we tested for protein-protein interactions between T4CPs and VirB10-like elements of different pT4SS. To the elements previously analysed from the cT4SS of plasmids R388, pKM101 and R6K (Llosa et al., 2003), we added those of the pT4SS VirB of At, Trw of Bt and VirB of Bs. The different T4CPs and VirB10s obtained from the pT4SS were expressed as fusion proteins with the T18 and T25 domains of adenylate cyclase (Cya). Cya domains were always placed at the $\mathrm{N}$-terminus, since both T4CPs and VirB10 homologues are expected to have their $\mathrm{N}$-termini in the cytoplasm, where the combined T18 + T25 can form an active Cya. Two-hybrid assays were performed with all pairs of proteins. Protein interactions, detected by development of blue colour on X-Gal-containing plates, were confirmed by measuring $\beta$-galactosidase activity.

Fig. 2 shows a summary of the results obtained. All interactions were made reciprocal (with each protein fused to the T25 or the T18 Cya domains) and no significant differences were found in any of the pairs (data not shown). The T4CP protein At VirD4 did not interact with any of the other fusion proteins. We do not have a way of testing the integrity of the protein, so no conclusions can be drawn from this negative result. The remaining constructions tested showed interactions with one another. VirB10 homologues interacted with themselves and with each other, as previously observed for the VirB10 members of cT4SS (Llosa et al., 2003). The main result was the existence of interactions between the conjugative T4CPs (TrwB, TraJ and TaxB) and the VirB10 components of pT4SS (At, $B t$ and $B s)$. Interactions with their cognate VirB10 homologues (TrwE, TraF and PilX10) are also shown for comparison, although in the absence of protein quantification the results are considered only qualitative.

We performed complementation tests of an R388 trwE mutant that is transfer-deficient (pSU4134; Table 2) with the various VirB10-like proteins used in the two-hybrid assays to further confirm the interactions detected in Fig. 2. Plasmids were constructed that carry the R388-trwE, $B t$-trwE and Bs-virB10 wild-type genes with appropriate expression signals under the control of the vector lac 


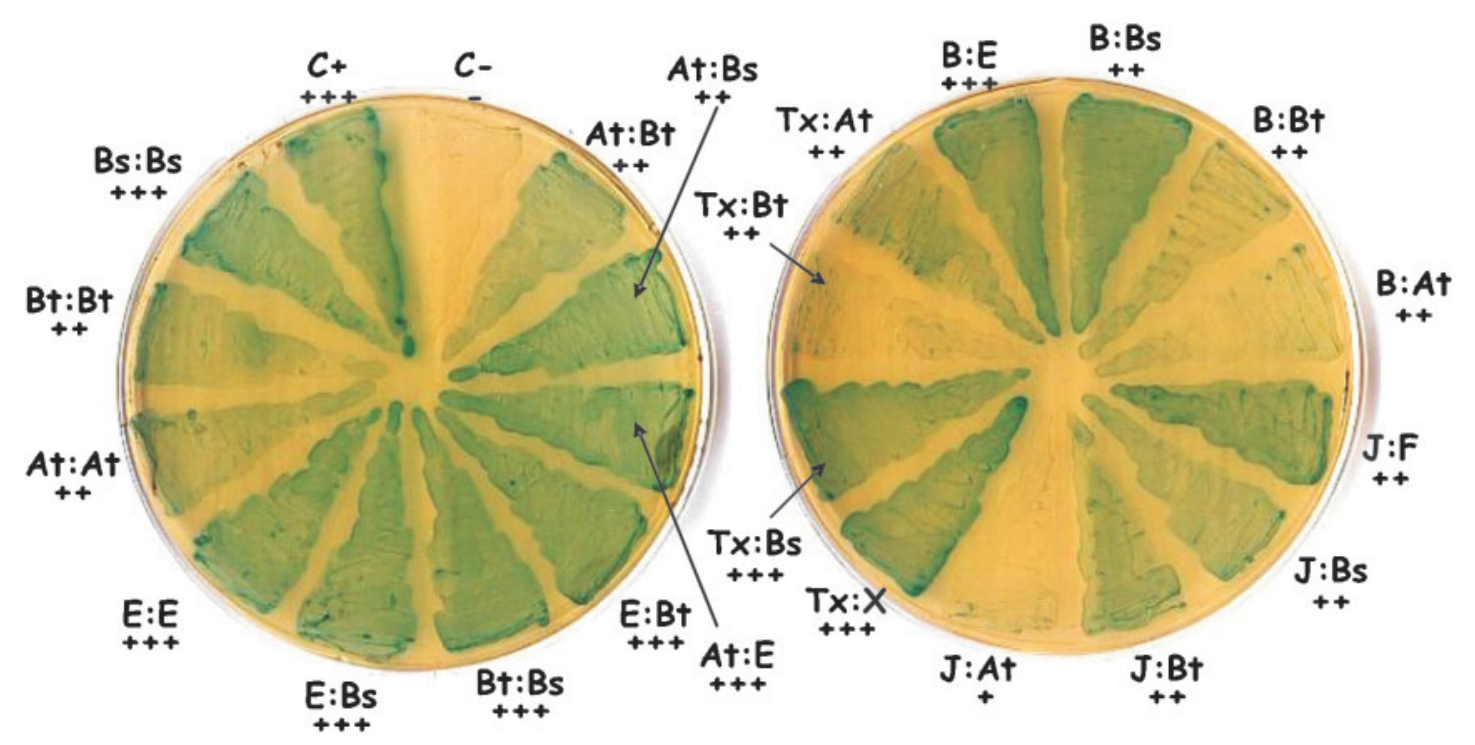

Fig. 2. Two-hybrid interactions among $\mathrm{T} 4 \mathrm{CP}$ and VirB10 homologues of various T4SS. Strain Ec DHM1 (cya) was transformed with plasmids encoding T25 and T18 fusions. Co-transformants were grown at $30{ }^{\circ} \mathrm{C}$ and spread on sections of LB plates supplemented with antibiotics and X-Gal for colour development. $\beta$-Galactosidase activity was calculated in Miller units and is summarized as follows:,$+++>1000$ units;,$++ 100-1000$ units;,$+ 20-100$ units;,$-<20$ units. Key plasmid pairs are shown in the figure and labelled in the format T18:T25 fusions with the following abbreviations: At, AtVirB10; Bs, Bs-VirB10; Bt, Bt-TrwE; B, R388-TrwB; E, R388-TrwE; J, pKM101-TraJ; F, pKM101-TraF; Tx, R6K-TaxB; X, R6K-PilX10; $\mathrm{C}+$ and $\mathrm{C}-$, positive and negative controls (T18zip:T25zip and T18:T25zip, respectively).

promoter (plasmids pHP102, pHP100 and pHP01; Table 2). The R388 trwE mutant was complemented by R388-TrwE in trans although the conjugation efficiency decreased more than one log compared to wild-type R388. The non-polar effect of the trwE insertion mutation was confirmed by complementation by a trwD mutant (pSU4063) to wild-type levels. The same frequency was obtained when matings were done in the presence of IPTG to increase trwE expression (data not shown). Since TrwE forms part of a multiprotein complex it might need to be co-expressed with other components of the complex for optimal efficiency. Requirement for tight regulation of the Bt-trwE copy number has been reported (Seubert et al., 2003).

With respect to heterologous complementations, we did not detect significant complementation with Bs-VirB10. However, there was a low but consistent complementation by Bt-TrwE, the closest homologue to R388-TrwE. The transfer efficiency of the R388 trwE mutant when complemented with $B t$-TrwE was 100 times lower than when complemented with R388-TrwE. Similar frequencies were obtained in the presence of IPTG (not shown). Thus, the interaction observed between R388-TrwB and Bt-TrwE could reflect a functional interaction since $B t$-TrwE can partially substitute for R388-TrwE.

\section{Analysis of the R388 Trw region}

The DNA sequence of the R388 T4SS genetic region (GenBank accession no. X81123) includes 11 genes, named
$\operatorname{trwN}$ to $\operatorname{tr} w D$, with homology to $\operatorname{virB1}$ to $\operatorname{virB11}$ respectively, plus four additional ORFs presumably involved in entry exclusion (eex) and regulation functions (korA, orf34 and korB) (Bolland et al., 1990 and our unpublished results). Fig. 1 shows a detailed map of this region. The role of $t r w N$ in R388 conjugation remains to be determined, since full transfer efficiency was obtained without this gene (Bolland et al., 1990). Analysis of the DNA sequence suggested that this region is organized in four operons: $\operatorname{kor} B$ (which is transcribed in the opposite direction to the rest of the genes), trwN-orf34, korA-trwI, and trwH-trwD. This assumption is based first on the arrangement of the genes within each proposed transcript, suggesting translational coupling, while two intergenic regions of more than $100 \mathrm{bp}$ are found between each proposed transcript (225 bp between $\operatorname{trwN}$ and korA, and 112 bp between trwI and trwH); second, on the presence of sequences with homology to the consensus promoter $5^{\prime}$ of each proposed transcript; and third, the three putative promoter regions are defined by the presence of 'kor boxes' (shown as dashed vertical lines in Fig. 1), presumed transcription control sites by the Kor proteins (R. Fernández, C. Revilla, M. P. Garcillán \& F. de la Cruz, unpublished), that are shared by the homologous T4SS of plasmid pKM101 and Bt-Trw (More et al., 1996; Seubert et al., 2003). From now on we will refer to the two putative operons containing the R388 T4SS genes as 'trw region 1' (korA-trwI) and 'trw region 2' (trwH-trwD).

In order to analyse this R388 region in detail, we obtained Tn5tacl insertion mutants along the R388 DNA segment 
Table 4. Selected Tn5tac1 insertion mutants in the R388 T4SS genetic region

\begin{tabular}{|c|c|c|c|c|c|c|c|c|}
\hline \multirow{2}{*}{$\begin{array}{l}\text { Insertion in } \\
\text { pSU4058 } \\
(\text { trwL-trwD) }\end{array}$} & \multirow{2}{*}{$\begin{array}{l}\text { Disrupted } \\
\text { gene }\end{array}$} & \multirow[t]{2}{*}{ Coords. ${ }^{*}$} & \multirow{2}{*}{$\begin{array}{l}\text { Same insertion } \\
\text { in } \mathbf{R} 388 \dagger\end{array}$} & \multicolumn{5}{|c|}{ Transfer frequency with helper plasmid $\ddagger$} \\
\hline & & & & None & $\begin{array}{l}\mathrm{pSU} 4058 \\
(\text { trwL-D) }\end{array}$ & $\begin{array}{c}\text { pHP109 } \\
(\text { trwH-D) }\end{array}$ & $\begin{array}{l}\text { pHP111 } \\
(\text { trwL-I) }\end{array}$ & $\begin{array}{l}\mathrm{pHP} 109+\mathrm{pHP} 111 \\
(\text { trwL-I+trwH-D) }\end{array}$ \\
\hline pSU4065 & $\operatorname{trwL}$ & 8931 & pSU4130 & $<10^{-6}$ & $2 \cdot 6 \times 10^{-1}$ & ND & $8 \cdot 6 \times 10^{-5}$ & $1 \cdot 0 \times 10^{-2}$ \\
\hline pSU4064 & $\operatorname{trwK}$ & 7271 & pSU4133 & $<10^{-6}$ & $8 \cdot 0 \times 10^{-2}$ & ND & $3 \cdot 2 \times 10^{-4}$ & $8 \cdot 4 \times 10^{-3}$ \\
\hline pSU4082 & trwJ & 5920 & pSU4136 & $<10^{-6}$ & $9 \cdot 0 \times 10^{-2}$ & ND & $5 \cdot 0 \times 10^{-2}$ & $1 \cdot 0 \times 10^{-2}$ \\
\hline \multirow[t]{2}{*}{ pSU4078 } & $\operatorname{trwH}$ & 3974 & pSU4131 & $5 \cdot 2 \times 10^{-5}$ & $1 \cdot 5 \times 10^{-1}$ & $1.6 \times 10^{-3}$ & ND & $1 \cdot 6 \times 10^{-2}$ \\
\hline & $\operatorname{trw} G$ & 3800 & pSU4035\$ & $<10^{-6}$ & $5 \cdot 1 \times 10^{-2}$ & ND & ND & ND \\
\hline pSU4070 & $\operatorname{trwF}$ & 2897 & pSU4135 & $<10^{-6}$ & $3 \cdot 0 \times 10^{-1}$ & $7 \cdot 0 \times 10^{-3}$ & ND & $2 \cdot 2 \times 10^{-2}$ \\
\hline pSU4067 & $\operatorname{trwE}$ & 1622 & pSU4134 & $<10^{-6}$ & $1 \cdot 8 \times 10^{-1}$ & $7 \cdot 9 \times 10^{-3}$ & ND & $9 \cdot 8 \times 10^{-3}$ \\
\hline pSU4063 & $\operatorname{trwD}$ & 622 & pSU4132 & $<10^{-6}$ & $1 \cdot 2 \times 10^{-1}$ & $9 \cdot 1 \times 10^{-3}$ & ND & $3.5 \times 10^{-2}$ \\
\hline
\end{tabular}

${ }^{*}$ Coordinates at the site of Tn5tac1 insertion (GenBank X81123). The transposon duplicates 9 bp upon insertion. The site of insertion has been considered $3^{\prime}$ to the 9 bp repeat closer to the $5^{\prime}$ end of the gene.

$\dagger$ Mutations were transferred to the R388 derivative pSU1425 by homologous recombination, as explained in Methods.

¥Each R388 insertion mutant was introduced into Ec D1210 with the indicated helper plasmids for complementation, and mated with Ec DH5 $\alpha$. Plasmid pSU4058 carries the complete trw region, and plasmids pHP109 and pHP111 carry putative operons 1 and 2, respectively (see text for further details). Transfer frequencies are expressed as number of transconjugants per donor cell; ND, not determined.

SpSU4035 is a $\Omega$ insertion mutant of pSU1087 (Table 2); the exact coordinates of the insertion were not determined. pSU4035 could not be assayed for complementation by pHP109 since both plasmids have a p15A replicon.

present in plasmid pSU4058, which includes trw regions 1 and 2. Tn5tacl insertions have been shown to be non-polar (Llosa et al., 1991), so mutations are expected to affect only the target ORF, unless high levels of expression of the gene are required for its normal functioning. Insertions were obtained in all trw genes except for $\operatorname{tr} w G$ and $\operatorname{tr} w M$. A trwG mutant was obtained from a previous collection of mutants by insertion of interposon $\Omega$ along the trw region (Bolland et al., 1990). All insertions affecting a trw gene conferred resistance to the pilus-specific phage PRD1. Selected insertions mapping in each gene (shown in Fig. 1) were transferred to R388 by homologous recombination to test for their conjugation frequency (see relevant plasmid pairs in Table 4). The $\operatorname{trw} G$ mutation was already in a plasmid containing the whole R388 transfer region. The nine mutants with insertions in different trw genes were transfer-deficient, except the mutant in $t r w H$, which conjugated about 1000-fold less efficiently than wild-type (Table 4). All were complemented to wild-type frequencies by pSU4058, which provides the whole korA-trwD region (Table 4). We confirmed the non-polar character of these mutants by showing full complementation of mutant pairs in adjacent genes in all cases (e.g. pSU4130 + pSU4064, see Table 4; data not shown).

We next dissected the T4SS region into two segments corresponding to trw regions 1 and 2 (plasmids pHP111 and pHP109, respectively; Fig. 1). In order to check for the integrity and functionality of both putative operons when expressed separately, we performed mobilization assays of plasmid pFJS134, containing the R388 oriT-trwABC region in a compatible vector. pFJS134 was mobilized from strain
Ec D1210 by plasmid pSU4058 (containing the korA-trwD region) at a frequency of $1 \cdot 1 \times 10^{-1}$ transconjugants per donor and it was mobilized with a similar frequency in the presence of plasmids $\mathrm{pHP} 109+\mathrm{pHP} 111$, providing trw regions 1 and $2\left(2 \cdot 1 \times 10^{-1}\right.$ transconjugants per donor), thus providing evidence that a functional T4SS is assembled when the two putative operons are expressed separately.

We also assayed complementation of the R388 insertion mutants by each putative trw operon separately, as shown in Table 4. All mutants were complemented to some extent; however, complementation levels were significantly lower than when complemented by pSU4058. When both pHP109 and pHP111 plasmids were present, complementation levels were higher, but still about 10 times lower than when complemented by pSU4058.

\section{Functional interactions between R388 cT4SS and BS pT4SS}

In order to detect possible functional relationships between the R388 and Brucella T4SS, a series of matings were performed using Bs strains as donors harbouring R388 or its mobilizable derivatives. R388 conjugated from $B s$ as efficiently as from $E c$ (about $10^{-1}$ transconjugants per donor). None of the R388 insertion mutants was mobilized from $B s$ significantly better than from $E c$ donors (data not shown), suggesting that individual T4SS components cannot be exchanged between the two T4SS. We did not detect conjugative transfer of a plasmid carrying the R388 oriT-trwABC region (pFJS134) through the intact Bs T4SS. We assayed mobilization of pFJS193, a plasmid containing 


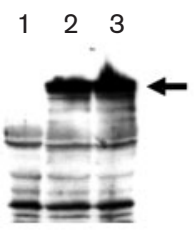

Anti-TrwC
12

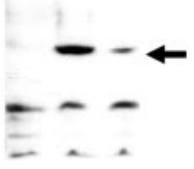

Anti-TrwD
123

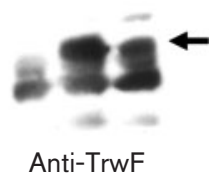

Fig. 3. Immunoblots showing Trw protein levels in cell extracts from Bs 1330 (sample 1), Bs 1330 (pSU2007) (sample 2) and Bs 1330 virB5::Kan (pSU2007) (sample 3). Sample preparation and Western blotting were performed as described in Methods. The primary antibody used is indicated at the bottom of each blot. Arrows indicate the expected size of the corresponding Trw protein.

the mobilization region of plasmid CloDF13, in order to test if Bs-T4SS could be used by a mobilizable plasmid rather than a conjugative system, since the former are more flexible in the use of T4SS. pFJS193 was not mobilized by the Bs T4SS either.

We assayed a Bs virB5 mutant that contains a polar mutation in virB5 (Boschiroli et al., 2002; O'Callaghan et al., 1999). Thus, in this strain presumably only the VirB1 to VirB4 proteins are produced and no functional T4SS is assembled. Consequently, the strain is avirulent in cellular models of infection (O'Callaghan et al., 1999). When R388 was mobilized from this strain, the transfer frequency dropped drastically to $10^{-6}$ transconjugants per donor. We determined R388 Trw protein levels in both wild-type and virB5 $B s$ strains harbouring R388. Fig. 3 shows immunoblots of cell extracts from $B s$ strains probed with anti-TrwC, antiTrwD and anti-TrwF antibodies. It can be observed that, while the amount of $\mathrm{TrwC}$ (the conjugative relaxase) remains constant in both wild-type and virB5 strains, the levels of the T4SS components TrwD and TrwF are diminished significantly. We calculated a 17 -fold decrease in the amount of TrwD in the Bs virB5 mutant compared to wild-type. Quantification of TrwF was not accurate due to non-specific binding of the anti-TrwF antibody to proteins of a size similar to TrwF (Fig. 3).

\section{Heterologous complementation between components of the R388 and Bt T4SS}

The Trw T4SS of R388 and Bt are the closest family members according to DNA sequence identity, yet they are a cT4SS and a pT4SS, respectively. Analyses of the functional relationships between these two T4SS are thus especially relevant. In previous sections we have shown that $B t-t r w E$ is the only VirB10 homologue capable of complementing a R388 trwE mutant. We extended this analysis with a series of complementation assays using plasmid pAB2, which carries the whole trw locus from $B t$ on a cosmid that can replicate in $E c$, and the R388 insertion mutants described in Table 4. Results are shown in Table 5. R388 derivatives with mutations in $\operatorname{tr} w D, \operatorname{trw} E, \operatorname{tr} w F, \operatorname{tr} w G$ and $\operatorname{tr} w H$ showed a
Table 5. Complementation of R388 T4SS mutants

Plasmids in the first column were introduced in Ec D1210 and mated with $E c$ DH5 $\alpha$. Plasmids pSU4130 to 4137 are described in Table 4.

\begin{tabular}{|c|c|c|c|}
\hline Plasmids in donor & R388 Trw & Bt T4SS & $\begin{array}{l}\text { Transfer } \\
\text { frequency }\end{array}$ \\
\hline pSU4130 & All but TrwL & - & $<10^{-6}$ \\
\hline pSU $4130+$ pAB2 & All but TrwL & + & $<10^{-6}$ \\
\hline pSU4133 & All but TrwK & - & $<10^{-6}$ \\
\hline pSU $4133+$ pAB2 & All but TrwK & + & $<10^{-6}$ \\
\hline pSU4136 & All but TrwJ & - & $<10^{-6}$ \\
\hline pSU $4136+$ pAB2 & All but TrwJ & + & $<10^{-6}$ \\
\hline pSU4137 & All but TrwI & - & $<10^{-6}$ \\
\hline pSU $4137+$ pAB2 & All but TrwI & + & $<10^{-6}$ \\
\hline pSU4131 & All but TrwH & - & $5 \cdot 2 \times 10^{-5}$ \\
\hline pSU $4131+$ pAB2 & All but TrwH & + & $1.6 \times 10^{-4}$ \\
\hline pSU4035 & All but TrwG & - & $<10^{-7 \star}$ \\
\hline pSU $4035+$ pAB2 & All but TrwG & + & $2 \cdot 9 \times 10^{-6}$ \\
\hline pSU4135 & All but TrwF & - & $<10^{-6}$ \\
\hline pSU $4135+$ pAB2 & All but TrwF & + & $1 \cdot 7 \times 10^{-5}$ \\
\hline pSU4134 & All but TrwE & - & $<10^{-6}$ \\
\hline pSU $4134+$ pAB2 & All but TrwE & + & $1 \cdot 1 \times 10^{-5}$ \\
\hline pSU4132 & All but TrwD & - & $<10^{-6}$ \\
\hline pSU4132+ pAB2 & All but TrwD & + & $2 \cdot 1 \times 10^{-2}$ \\
\hline pSU1423† + pSU 4058 & TrwABC + TrwL-D & - & $4 \times 10^{-1}$ \\
\hline $\mathrm{pSU} 1423 \dagger+\mathrm{pAB} 2$ & TrwABC & + & $<10^{-6}$ \\
\hline $\begin{array}{l}\text { pSU1423†+ } \\
\text { pHP111 + pAB2 }\end{array}$ & TrwABC + TrwL-I & + & $<10^{-6}$ \\
\hline $\begin{array}{l}\text { pSU4051†+ } \\
\text { pHP109 + pAB2 }\end{array}$ & TrwABC + TrwH-D & + & $<10^{-6}$ \\
\hline
\end{tabular}

${ }^{\star}$ Matings were scaled up to better discriminate between a low transfer frequency and the negative control.

$\dagger$ Plasmids pSU1423 and pSU4051 contain the same R388 region, but in different replicons (see Table 2).

significant increase in transfer efficiency in the presence of plasmid pAB2, while those with mutations in trwI, trwJ, trwK and trwL remained transfer-deficient. In order to rule out the possibility that recombination had taken place between the two trw regions, transconjugants from the above matings were checked for tetracycline sensitivity and were also used as donors in a new round of conjugations; the results confirmed that transconjugants remained transfer-deficient.

We next tried to mobilize an R388 derivative by the Bt-T4SS (Table 5). Plasmid pSU1423 carries R388 oriT + trwABC and could be readily mobilized by a plasmid containing trwL to trwD (pSU4058). However, we could not detect mobilization of plasmid pSU1423 by the Bt-trw locus present in plasmid pAB2. Since complementation of R388 single mutants by pAB2 was observed only for mutations lying in the trwH-trwD putative operon, plasmids containing ori $T+\operatorname{tr} w A B C$ were assayed for mobilization by $\mathrm{pAB} 2$ in the presence of an additional plasmid carrying one of the 
two R388 trw regions (either pHP111 or pHP109). The pAB2 plasmid did not provide the remaining functions in either case (Table 5).

\section{DISCUSSION}

In this work we have analysed the functional interactions between cT4SS and pT4SS in order to better understand their constituent genetic modules and the level of functional conservation among their individual protein components.

Our model cT4SS is the R388 Trw system. Its T4SS genetic determinant comprises genes trwL-trwD (Fig. 1). The adjacent gene $\operatorname{trw} N$ is a homologue of the VirB1-type components of other T4SS; however, to date there is no evidence that trwN is required for R388 conjugal transfer (Bolland et al., 1990), so we have excluded this gene from our analysis. Non-polar insertion mutations proved that genes $\operatorname{trwD}, E, F, G, I, J, K$ and $L$ are essential for R388 conjugation; an insertion in trwH renders a plasmid that can self-transfer with very low efficiency (Table 4). Analysis of the DNA sequence strongly suggests that the trwL-D genes are expressed from two transcriptional units: korA-trwI and trwH-trwD. The functionality of the promoter upstream of trwH has been shown for the homologous regions in BtTrw and conjugative plasmid pKM101 (More et al., 1996; Seubert et al., 2003). We have shown that expression of each putative operon from different replicons allows the assembly of a functional T4SS, as tested by high-efficiency mobilization of a plasmid carrying R388 oriT-trwABC (pFJS134).

It was previously reported that conjugative $\mathrm{T} 4 \mathrm{CPs}$ could interact with VirB10 homologues from other cT4SS, and moreover the strength of this interaction affected the efficiency of DNA mobilization (Llosa et al., 2003). We extended the analysis of this interaction to the pT4SS of $A t, B t$ and $B s$. By two-hybrid analysis we have shown that conjugative T4CPs interact similarly with VirB10 homologues from pT4SS. VirB10 proteins interact with themselves and with each other, from both pT4SS and cT4SS. Thus, these interactions are conserved even in T4SS that do not have any known T4CP, such as $B t$ and $B s$. In the case of $B t$, it cannot be excluded that the T4CP from the VirB T4SS is being used by both coexisting T4SS (Schröder \& Dehio, 2005).

The interaction detected between TrwB and Bt-TrwE (the closest TrwE homologue according to the amino acid sequence) may also reflect a functional interaction since $B t$ TrwE could partially complement an R388-trwE mutation. This complementation adds evidence to previous work showing that Bt-trwD and Bt-trwH could complement trwD and trwH mutations in R388 (Seubert et al., 2003), underscoring the functional similarity between the R388and Bt-Trw T4SS. This prompted us to extend the complementation analysis between these two systems. As shown in Table 5, several R388 trw mutants could conjugate at low efficiency in the presence of pAB2, a cosmid providing the whole Bt-trw region. It is noteworthy that complementation is observed only for genes belonging to R388 trw region 2 . The conservation of gene synteny is frequently due to the need for co-expression of gene products that strongly depend on each other for function. Region 2 encodes the more conserved elements of the T4SS apparatus, the 'core' components, which may play a similar function in both systems and could thus be exchanged to a certain extent. On the other hand, the T4SS components encoded in region 1 may be system-specific. For instance, the pilus components (TrwL and TrwJ) could be responsible for specific interactions with the recipient cells.

Possible functional relationships between R388-Trw and BsVirB T4SS were also addressed. Bs T4SS did not complement any of the R388 T4SS individual mutants, not surprisingly considering that the similarity between these two T4SS is significantly lower than that between the R388- and Bt-Trw T4SS. However, when a Bs virB5 polar mutant was used as a donor, a strong dominant negative effect was exerted on R388 transfer. Dominant negative effects are typical of proteins that make oligomers. The results suggest that when components of the Bs-T4SS cannot assemble into their own T4SS, they interact with the related R388 Trw proteins, resulting in non-functional heteromultimers. The unassembled Trw proteins are probably destabilized, leading to diminished cellular levels of the R388 T4SS proteins (Fig. 3). The poisoning effect could be mediated for example by VirB2, the homologue to the major pilus component. Pili are made up of a high number of pilin subunits and interference by a low number of heterologous pilin subunits could impede T4SS assembly, thus explaining the strong interference observed.

The existence of interactions between cT4SS and pT4SS components opens up the attractive possibility that DNA substrates recruited by the T4CPs could be coupled to pT4SS, so pathogens could be used as intracellular DNA delivery tools. So far, our attempts to use the pT4SS of both $B s$ and Bt to mobilize R388 derivatives lacking their cognate T4SS have had no success. Substrate selection by T4SS probably depends on more than a single protein-protein interaction. Each T4SS component interacts with several other components of the secretion machinery (Ward et al., 2002). Recent results on the At T4SS show that formation of the VirB9-VirB10 complex is essential for T-DNA substrate selection and translocation through the distal portion of the secretion channel (Cascales \& Christie, 2004a, b; Jakubowski et al., 2005). The partial functional exchanges found between the R388 and Bt T4SS open up the way for mutagenesis experiments in order to obtain mutant T4CPs that better interact with selected pT4SS components or subassemblies. The study of these two highly related T4SS will also help in understanding the similarities and differences between cT4SS and pT4SS.

\section{ACKNOWLEDGEMENTS}

We are grateful to Ana M. Cayón and Jorge Díaz for technical assistance, to Dr David O'Callaghan (INSERM, France) for providing 
strains Bs 1330 and Bs 1330 virB5, and to Dr Iñaki Sastre for providing anti-TrwF antibody. Work in the authors' laboratories was supported by grants BIO2002-00063, BMC2002-00379 and BIO2004-06167 from the MEC (Spain) to M.L., F.C. and F. J.S., respectively; by grant QLK-CT-2001-01200 from the EC to J.M.G.; and by grant API04/09 from Fundación Marqués de Valdecilla to F. J.S. H.P. was supported by a predoctoral fellowship from Fundación Marqués de Valdecilla.

\section{REFERENCES}

Alegria, M. C., Souza, D. P., Andrade, M. O., Docena, C., Khater, L., Ramos, C. H., da Silva, A. C. \& Farah, C. S. (2005). Identification of new protein-protein interactions involving the products of the chromosome- and plasmid-encoded type IV secretion loci of the phytopathogen Xanthomonas axonopodis pv. citri. J Bacteriol 187, 2315-2325.

Atmakuri, K., Ding, Z. \& Christie, P. J. (2003). VirE2, a type IV secretion substrate, interacts with the VirD4 transfer protein at cell poles of Agrobacterium tumefaciens. Mol Microbiol 49, 1699-1713.

Atmakuri, K., Cascales, E. \& Christie, P. J. (2004). Energetic components VirD4, VirB11 and VirB4 mediate early DNA transfer reactions required for bacterial type IV secretion. Mol Microbiol 54, 1199-1211.

Baron, C., Thorstenson, Y. R. \& Zambryski, P. C. (1997). The lipoprotein VirB7 interacts with VirB9 in the membranes of Agrobacterium tumefaciens. J Bacteriol 179, 1211-1218.

Bartolomé, B., Jubete, Y., Martínez, E. \& de la Cruz, F. (1991). Construction and properties of a family of pACYC184-derived cloning vectors compatible with pBR322 and its derivatives. Gene 102, 75-78.

Beaupre, C. E., Bohne, J., Dale, E. M. \& Binns, A. N. (1997). Interactions between VirB9 and VirB10 membrane proteins involved in movement of DNA from Agrobacterium tumefaciens into plant cells. J Bacteriol 179, 78-89.

Berger, B. R. \& Christie, P. J. (1994). Genetic complementation analysis of the Agrobacterium tumefaciens virB operon: virB2 through virB11 are essential virulence genes. J Bacteriol 176, 3646-3660.

Bolland, S., Llosa, M., Avila, P. \& de la Cruz, F. (1990). General organization of the conjugal transfer genes of the IncW plasmid R388 and interactions between R388 and IncN and IncP plasmids. J Bacteriol 172, 5795-5802.

Boschiroli, M. L., Ouahrani-Bettache, S., Foulongne, V. \& 7 other authors (2002). The Brucella suis virB operon is induced intracellularly in macrophages. Proc Natl Acad Sci U S A 99, 1544-1549.

Boyer, H. W. \& Roulland-Dussoix, D. (1969). A complementation analysis of the restriction and modification of DNA in Escherichia coli. J Mol Biol 41, 459-472.

Bruce, K. D. \& Jordens, J. Z. (1991). Characterization of noncapsulate Haemophilus influenzae by whole-cell polypeptide profiles, restriction endonuclease analysis, and rRNA gene restriction patterns. J Clin Microbiol 29, 291-296.

Cabezón, E., Sastre, J. I. \& de la Cruz, F. (1997). Genetic evidence of a coupling role for the TraG protein family in bacterial conjugation. Mol Gen Genet 254, 400-406.

Cascales, E. \& Christie, P. J. (2003). The versatile bacterial Type IV secretion systems. Nat Rev Microbiol 1, 137-149.

Cascales, E. \& Christie, P. J. (2004a). Definition of a bacterial type IV secretion pathway for a DNA substrate. Science 304, 1170-1173.
Cascales, E. \& Christie, P. J. (2004b). Agrobacterium VirB10, an ATP energy sensor required for type IV secretion. Proc Natl Acad Sci U S A 101, 17228-17233.

Chow, W. Y. \& Berg, D. E. (1988). Tn5tacl, a derivative of transposon Tn5 that generates conditional mutations. Proc Natl Acad Sci U S A 85, 6468-6472.

Das, A. \& Xie, Y. H. (2000). The Agrobacterium T-DNA transport pore proteins VirB8, VirB9, and VirB10 interact with one another. $J$ Bacteriol 182, 758-763.

Das, A., Anderson, L. B. \& Xie, Y. H. (1997). Delineation of the interaction domains of Agrobacterium tumefaciens VirB7 and VirB9 by use of the yeast two-hybrid assay. J Bacteriol 179, 3404-3409.

Farizo, K. M., Cafarella, T. G. \& Burns, D. L. (1996). Evidence for a ninth gene, $p t l I$, in the locus encoding the pertussis toxin secretion system of Bordetella pertussis and formation of a PtII-PtlF complex. J Biol Chem 271, 31643-31649.

Gomis-Rüth, F. X., Moncalián, G., Pérez-Luque, R., González, A., Cabezón, E., de la Cruz, F. \& Coll, M. (2001). The bacterial conjugation protein TrwB resembles ring helicases and F1-ATPase. Nature 409, 637-641.

Grandoso, G., Llosa, M., Zabala, J. C. \& de la Cruz, F. (1994). Purification and biochemical characterization of TrwC, the helicase involved in plasmid R388 conjugal DNA transfer. Eur J Biochem 226, 403-412.

Grant, S. G., Jessee, J., Bloom, F. R. \& Hanahan, D. (1990). Differential plasmid rescue from transgenic mouse DNAs into Escherichia coli methylation-restriction mutants. Proc Natl Acad Sci U S A 87, 4645-4649.

Hapfelmeier, S., Domke, N., Zambryski, P. C. \& Baron, C. (2000). VirB6 is required for stabilization of VirB5 and VirB3 and formation of VirB7 homodimers in Agrobacterium tumefaciens. J Bacteriol 182, 4505-4511.

Hoppner, C., Liu, Z., Domke, N., Binns, A. N. \& Baron, C. (2004). VirB1 orthologs from Brucella suis and pKM101 complement defects of the lytic transglycosylase required for efficient type IV secretion from Agrobacterium tumefaciens. J Bacteriol 186, 1415-1422.

Jakubowski, S. J., Krishnamoorthy, V., Cascales, E. \& Christie, P. J. (2004). Agrobacterium tumefaciens VirB6 domains direct the ordered export of a DNA substrate through a type IV secretion. Syst J Mol Biol 341, 961-977.

Jakubowski, S. J., Cascales, E., Krishnamoorthy, V. \& Christie, P. J. (2005). Agrobacterium tumefaciens VirB9, an outer-membraneassociated component of a Type IV secretion system, regulates substrate selection and T-pilus biogenesis. J Bacteriol 187, 3486-3495.

Karimova, G., Pidoux, J., Ullmann, A. \& Ladant, D. (1998). A bacterial two-hybrid system based on a reconstituted signal transduction pathway. Proc Natl Acad Sci U S A 95, 5752-5756.

Karimova, G., Ullmann, A. \& Ladant, D. (2001). Protein-protein interaction between Bacillus stearothermophilus tyrosyl-tRNA synthetase subdomains revealed by a bacterial two-hybrid system. J Mol Microbiol Biotechnol 3, 73-82.

Karimova, G., Dautin, N. \& Ladant, D. (2005). Interaction network among Escherichia coli membrane proteins involved in cell division as revealed by bacterial two-hybrid analysis. J Bacteriol 187, 2233-2243.

Koraimann, G. (2003). Lytic transglycosylases in macromolecular transport systems of Gram-negative bacteria. Cell Mol Life Sci 60, 2371-2388.

Krall, L., Wiedemann, U., Unsin, G., Weiss, S., Domke, N. \& Baron, C. (2002). Detergent extraction identifies different VirB protein subassemblies of the type IV secretion machinery in the membranes of Agrobacterium tumefaciens. Proc Natl Acad Sci U S A 99, 11405-11410 
Kumar, R. B., Xie, Y. H. \& Das, A. (2000). Subcellular localization of the Agrobacterium tumefaciens T-DNA transport pore proteins: VirB8 is essential for the assembly of the transport pore. Mol Microbiol 36, 608-617.

Kushner, S. R., Nagaishi, H., Templin, A. \& Clark, A. J. (1971). Genetic recombination in Escherichia coli: the role of exonuclease I. Proc Natl Acad Sci U S A 68, 824-827.

Lai, E. M. \& Kado, C. I. (1998). Processed VirB2 is the major subunit of the promiscuous pilus of Agrobacterium tumefaciens. J Bacteriol 180, 2711-2717.

Llosa, M. \& de la Cruz, F. (2005). Bacterial conjugation: a potential tool for genomic engineering. Res Microbiol 156, 1-6.

Llosa, M. \& O'Callaghan, D. (2004). Euroconference on the biology of Type IV secretion processes: bacterial gates into the outer world. Mol Microbiol 53, 1-8.

Llosa, M., Jubete, Y. \& de la Cruz, F. (1991). Tn5tacl insertion polarity in Escherichia coli. Plasmid 26, 222-224.

Llosa, M., Bolland, S. \& de la Cruz, F. (1994). Genetic organization of the conjugal DNA processing region of the IncW plasmid R388. J Mol Biol 235, 448-464.

Llosa, M., Zunzunegui, S. \& de la Cruz, F. (2003). Conjugative coupling proteins interact with cognate and heterologous VirB10-like proteins while exhibiting specificity for cognate relaxosomes. Proc Natl Acad Sci U S A 100, 10465-10470.

Martínez, E. \& de la Cruz, F. (1988). Transposon Tn21 encodes a RecA-independent site-specific integration system. Mol Gen Genet 211, 320-325.

Miller, J. H. (1992). A Short Course in Bacterial Genetics : a Laboratory Manual and Handbook for Escherichia coli and Related Bacteria. Cold Spring Harbor, NY: Cold Spring Harbor Laboratory.

Moncalián, G., Grandoso, G., Llosa, M. \& de la Cruz, F. (1997). oriTprocessing and regulatory roles of TrwA protein in plasmid R388 conjugation. J Mol Biol 270, 188-200.

More, M. I., Pohlman, R. F. \& Winans, S. C. (1996). Genes encoding the pKM101 conjugal mating pore are negatively regulated by the plasmidencoded KorA and KorB proteins. J Bacteriol 178, 4392-4399.

Núñez, B. \& de la Cruz, F. (2001). Two atypical mobilization proteins are involved in plasmid CloDF13 relaxation. Mol Microbiol 39, 1088-1099.

O'Callaghan, D., Cazevieille, C., Allardet-Servent, A., Boschiroli, M. L., Bourg, G., Foulongne, V., Frutos, P., Kulakov, Y. \& Ramuz, M. (1999). A homologue of the Agrobacterium tumefaciens VirB and Bordetella pertussis Ptl type IV secretion systems is essential for intracellular survival of Brucella suis. Mol Microbiol 33, 1210-1220.

Rivas, S., Bolland, S., Cabezón, E., Goñi, F. M. \& de la Cruz, F. (1997). TrwD, a protein encoded by the IncW plasmid R388, displays an ATP hydrolase activity essential for bacterial conjugation. J Biol Chem 272, 25583-25590.
Rouot, B., Alvarez-Martinez, M. T., Marius, C. \& 7 other authors (2003). Production of the type IV secretion system differs among Brucella species as revealed with VirB5- and VirB8-specific antisera. Infect Immun 71, 1075-1082.

Sadler, J. R., Tecklenburg, M. \& Betz, J. L. (1980). Plasmids containing many tandem copies of a synthetic lactose operator. Gene 8, 279-300.

Sambrook, J., Fritsch, E. F. \& Maniatis, T. (1989). Molecular Cloning: a Laboratory Manual, 2nd edn. Cold Spring Harbor, NY: Cold Spring Harbor Laboratory.

Sastre, J. I. (1996). El extremo carboxilo de la proteína TraD del plásmido $F$ confiere especificidad y eficiencia en el proceso de la conjugación. PhD Thesis, Universidad de Cantabria.

Schmidt-Eisenlohr, H., Domke, N. \& Baron, C. (1999). TraC of IncN plasmid pKM101 associates with membranes and extracellular high-molecular-weight structures in Escherichia coli. J Bacteriol 181, 5563-5571.

Schröder, G. \& Dehio, C. (2005). Virulence-associated type IV secretion systems of Bartonella. Trends Microbiol 13, 336-342.

Seubert, A., Hiestand, R., de la Cruz, F. \& Dehio, C. (2003). A bacterial conjugation machinery recruited for pathogenesis. Mol Microbiol 49, 1253-1266.

Shamaei-Tousi, A., Cahill, R. \& Frankel, G. (2004). Interaction between protein subunits of the type IV secretion system of Bartonella henselae. J Bacteriol 186, 4796-4801.

Spudich, G. M., Fernandez, D., Zhou, X. R. \& Christie, P. J. (1996) Intermolecular disulfide bonds stabilize VirB7 homodimers and VirB7/VirB9 heterodimers during biogenesis of the Agrobacterium tumefaciens T-complex transport apparatus. Proc Natl Acad Sci U S A 93, 7512-7517.

Stewart, G. S., Lubinsky-Mink, S., Jackson, C. G., Cassel, A. \& Kuhn, J. (1986). pHG165: a pBR322 copy number derivative of pUC8 for cloning and expression. Plasmid 15, 172-181.

Tato, I., Zunzunegui, S., de la Cruz, F. \& Cabezón, E. (2005). TrwB, the coupling protein involved in DNA transport during bacterial conjugation, is a DNA-dependent ATPase. Proc Natl Acad Sci U S A 102, 8156-8161.

Towbin, H., Staehelin, T. \& Gordon, J. (1979). Electrophoretic transfer of proteins from polyacrylamide gels to nitrocellulose sheets: procedure and some applications. Proc Natl Acad Sci U S A 76, 4350-4354.

Vergunst, A. C., Schrammeijer, B., den Dulk-Ras, A., de Vlaam, C. M., Regensburg-Tuink, T. J. \& Hooykaas, P. J. (2000). VirB/D4dependent protein translocation from Agrobacterium into plant cells. Science 290, 979-982.

Ward, D. V., Draper, O., Zupan, J. R. \& Zambryski, P. C. (2002). Peptide linkage mapping of the Agrobacterium tumefaciens virencoded type IV secretion system reveals protein subassemblies. Proc Natl Acad Sci U S A 99, 11493-11500. 